
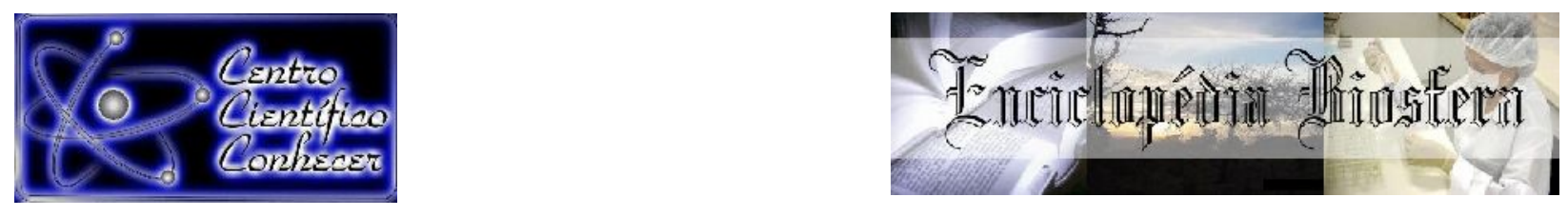

\title{
MÉTODOS ECODOPPLERCARDIOGRÁFICOS PARA AVALIAÇÃO DA FUNÇÃO SISTÓLICA DO VENTRÍCULO ESQUERDO EM CÃES
}

Paulo Roberto de Sousa', Tayanne Gobbi Mendes ${ }^{2}$, Rosângela de oliveira Alves Carvalho $^{3}$

1 - Médico Veterinário, Mestrando em Ciência Animal, Escola de Veterinária e

Zootecnica da Universidade Federal de Goiás, Goiânia - GO, Brasil. E-mail: paulo_rsousa@hotmail.com

2 - Médica Veterinária, Mestranda em Ciência Animal, Escola de Veterinária e Zootecnica da Universidade Federal de Goiás, Goiânia - GO, Brasil.

3 - Profa dra do Programa de Pós-graduação em Ciência Animal da Escola de

Veterinária e Zootecnia da Universidade Federal de Goiás, Goiânia - GO, Brasil.

Recebido em: 06/04/2019 - Aprovado em: 10/06/2019 - Publicado em: 30/06/2019 DOI: 10.18677/EnciBio_2019A89

\begin{abstract}
RESUMO
O exame ecodopplercardiográfico é empregado rotineiramente na clínica de pequenos animais, sendo considerado o método diagnóstico não invasivo de escolha quando há suspeita de qualquer cardiopatia, pois permite identificar alterações cardíacas estruturais e avaliar a função miocárdica. O estudo da função sistólica do ventrículo esquerdo (VE) representa uma das mais importantes indicações do exame ecodopplercardiográfico, tendo à disposição vários índices que podem ser obtidos por diferentes métodos. Dentre as dificuldades em determinar com precisão a função sistólica, destacam-se as cardiopatias que acarretam sobrecarga de volume e outras alterações hemodinâmicas que afetam diretamente os índices ecodopplercardiográficos utilizados na avaliação ventricular. A disfunção sistólica é considerada como um indicador de mau prognóstico, o que na medicina veterinária é uma das principais preocupações na avaliação dos pacientes cardiopatas, pois a presença da disfunção ventricular cursa com alterações miocárdicas estruturais e funcionais contribuindo para o desenvolvimento da insuficiência cardíaca (IC), que representa na maioria das vezes a via final da doença cardíaca. Assim a utilização correta da ecodopplercardiografia requer do médico veterinário o conhecimento prévio dos índices disponíveis para determinar a função sistólica.
\end{abstract}

PALAVRAS-CHAVE: Cães, Ecodopplercardiografia, função sistólica.

\section{ECODOPERCARDIOGRAPHIC METHODS FOR EVALUATION OF THE LEFT VENTRICULAR SYSTEMS FUNCTION IN DOGS}

\begin{abstract}
Doppler echocardiography is routinely used in the small animal clinic, being considered the noninvasive diagnostic method of choice when any cardiopathy is suspected, since it allows the identification of structural cardiac changes and the evaluation of myocardial function. The study of the left ventricular (LV) systolic
\end{abstract}


function represents one of the most important indications of the Doppler echocardiography exam, with several indexes available that can be obtained by different methods. Among the difficulties in accurately determining the systolic function, we highlight the cardiopathies that cause volume overload and other hemodynamic changes that directly affect the Doppler echocardiographic index used in the ventricular evaluation. Systolic dysfunction is considered an indicator of poor prognosis, which in veterinary medicine is one of the main concerns in the evaluation of patients with heart disease, since the presence of ventricular dysfunction presents with structural and functional myocardial alterations contributing to the development of heart failure (HF), which most often represents the ultimate pathway of heart disease. Thus, correct use of Doppler echocardiography requires prior knowledge of the indexes available to determine systolic function.

KEYWORDS: Echodopplercardiography, systolic function, dogs.

\section{INTRODUÇÃO}

A função sistólica do VE é caracterizada pela capacidade do coração em ejetar sangue para a circulação sistêmica após cada batimento cardíaco e, a disfunção sistólica é descrita como a incapacidade de bombeamento realizado pelo ventrículo acompanhada da diminuição na fração de ejeção (FE) (SARGENT et al., 2015; CHETBOUL et al., 2016).

Dentre os métodos ecodopplercardiográficos convencionais mais utilizados para determinar a função sistólica do VE, estão a fração de encurtamento (FS) e FE (BONAGURA; SCHOBER, 2009). Várias outras técnicas são utilizadas para determinar a função ventricular sistólica, tais como: volumes ventriculares, análise do VE em modo-B e modo-M, doppler espectral, doppler tecidual, ST e STR (CHETBOUL et al., 2016).

Para melhor compreensão do estudo ecodopplercardiográfico aplicado à análise da função sistólica do VE em cães, esta revisão inicia-se pela descrição anatômica e geométrica do VE e sobre a fisiologia das fases que compõem a sístole ventricular. Posteriormente, serão descritas as técnicas ecocopplercardiográficas disponíveis para avaliar a sístole ventricular.

\section{Anatomia e geometria ventricular esquerda}

O coração é um órgão que está situado no interior da cavidade torácica, no mediastino médio, entre as cavidades pleurais, possui quatro câmaras, dois átrios e dois ventrículos, separadas entre si pelas valvas atrioventriculares mitral e tricúspide. Longitudinalmente, os átrios estão separados pelo septo interatrial e os ventrículos pelo septo interventricular (CHETBOUL et al., 2016).

O coração na espécie canina está representado como um músculo cardíaco composto por três camadas: interna ou endocárdio, que delimita a cavidade dos átrios e ventrículos, média ou miocárdio que compreende o músculo cardíaco e, por fim, a externa ou epicárdio, que é a parte mais externa do coração (LAVALLE; RENDON, 2015).

Conceitos modernos de anatomia cardíaca estão firmados nas descobertas realizadas pelo médico cardiologista espanhol Francisco TorrentGuasp, que a partir de estudos em anatomia cardíaca reportou a teoria da banda miocárdica ventricular helicoidal (SALAMANCA et al., 2015). Esta teoria descreve o miocárdio ventricular como uma única banda muscular contínua, tendo origem na 
raiz da artéria pulmonar com término na artéria aorta, tomando forma de espiral em uma configuração helicoidal (LAVALLE; RENDON, 2015).

Estudos anatômicos do VE em humanos e cães tem demonstrado a existência de fibras miocárdicas orientadas em sentido radial e longitudinal, tendo maior predomínio de fibras radiais na base do VE com redução em sentido apical, sendo que estas fibras ocupam principalmente a camada média da parede ventricular, ao contrário das fibras longitudinais que estão mais presentes nas camadas internas e externas (Figura 1) (CHETBOUL et al., 2016).

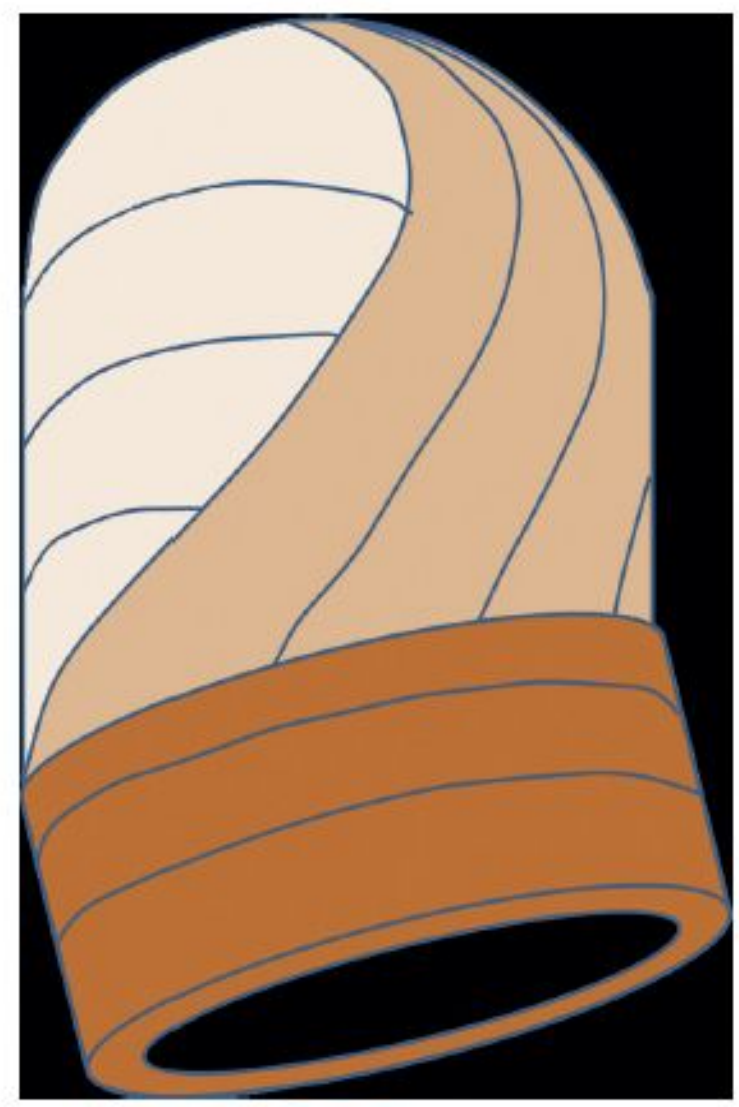

FIGURA 1 - Observar orientação das fibras miocárdicas do VE. Maior predomínio das fibras radiais na base do VE e fibras longitudinais distribuídas em maior quantidade na região apical. Fonte: Chetboul et al. (2016)

O VE apresenta cinética complexa, que resultada em três tipos de movimentos a partir do arranjo anatômico das fibras miocárdicas, estes incluem: movimentos radiais, circunferenciais e longitudinais (Figuras 2 e 3) (CHETBOUL et al., 2016; SURESH, 2016). 


\section{Sístole}

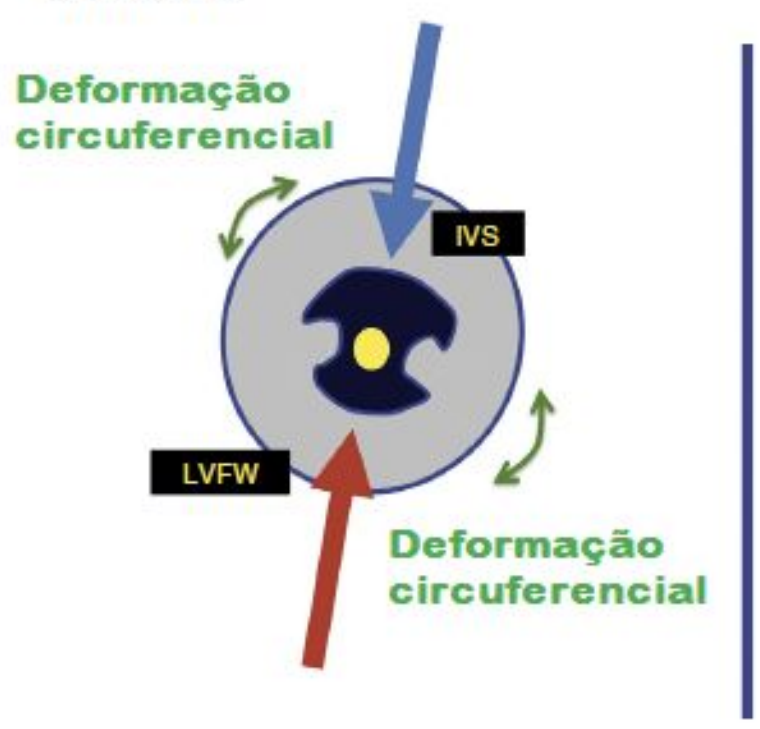

Diástole

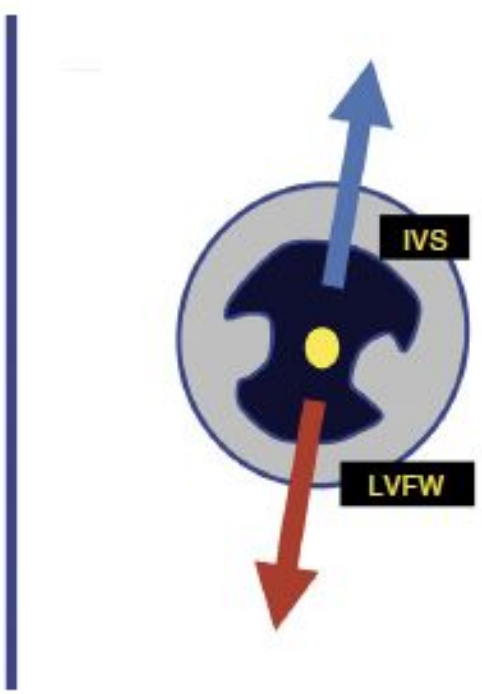

FIGURA 2 - Representação do movimento radial do VE. Na sístole, a parede livre do VE (LVFW) e o SIV (IVS) se convergem para um centro geométrico (ponto amarelo) situado no centro da cavidade ventricular, contudo estas mesmas paredes se divergem durante a diástole. Há também deformação circunferencial com redução das fibras durante a sístole.

FONTE: Adaptado de Chetboul et al. (2016) 


\section{Sístole}

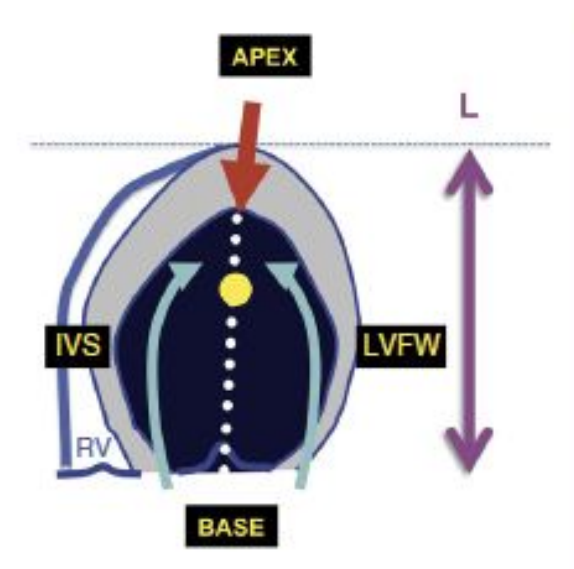

Diástole

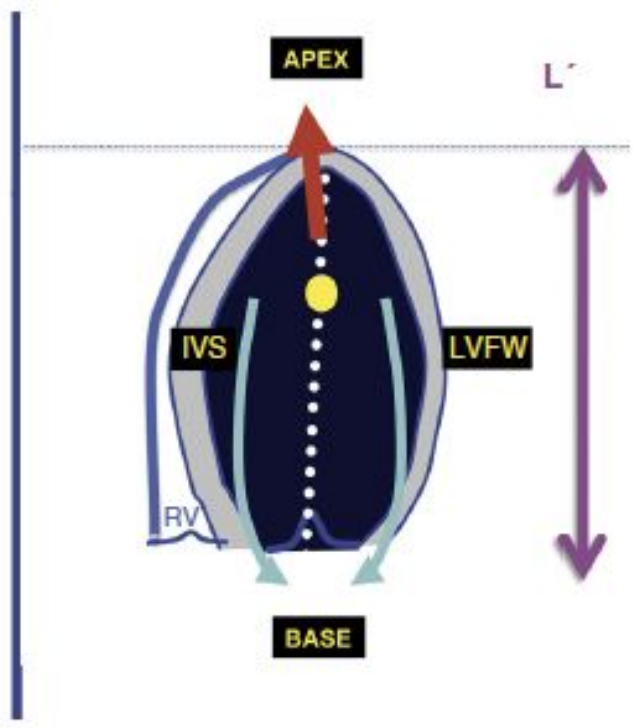

FIGURA 3 - Representação do movimento longitudinal do VE. Na sístole o comprimento do coração se reduz, em que o ápice (APEX), a parede livre do VE (LVFW) e o septo interventricular (IVS) convergem em direção ao centro geométrico (ponto amarelo), por outro lado, no período de diástole estas três paredes se divergem com um movimento do ápice oposto a outras duas paredes.

FONTE: Adaptado de Chetboul et al. (2016)

\section{Fases da sístole}

O ciclo cardíaco ou batimento cardíaco é formado por duas fases, uma diastólica ou de enchimento ventricular e outra sistólica ou ejeção ventricular. $\mathrm{Na}$ fase sistólica o VE tem como objetivo ejetar o volume de sangue em seu interior, proveniente das veias pulmonares e átrio esquerdo, através da artéria aorta em sentido à circulação sistêmica (POLLOCK; MAKARYUS, 2018).

O período sistólico é composto por uma sequência de três fases: contração isovolumétrica, ejeção ventricular rápida e ejeção ventricular lenta. A fase de contração isovolumétrica representa o início da sístole ventricular, e inicia-se logo após o registro do complexo QRS pelo eletrocardiograma, tendo neste momento as valvas atrioventriculares e semilunares completamente fechadas, então inicia o período de contração ventricular como resposta ao estímulo elétrico, evento que gera aumento abrupto da pressão intraventricular e faz com que a valva aórtica se abra, iniciando a fase de ejeção ventricular rápida, pois a pressão no interior do VE excede os valores de pressão na aorta fazendo com que esta se abra e permita a ejeção ventricular. Por fim, ocorre a fase de ejeção ventricular lenta, em que há queda da pressão no interior do VE abaixo da pressão aórtica, porém o fluxo através da aorta continua devido à energia cinética que faz impulsionar o sangue no sentido 
da circulação sistêmica até que a pressão seja mínima, promovendo o fechamento da valva aórtica e encerrando a fase sistólica (KLABUNDE, 2012).

\section{Avaliação por ecodopplercardiografia}

O exame ecodopplercardiográfico aplicado ao estudo da função ventricular fornece informações valiosas das condições hemodinâmicas do paciente cardiopata (BOON, 2011, SARGENT et al., 2015). Uma das principais aplicações desta ferramenta é o estudo da função do VE, que apresenta vários índices ecocardiográficos com o propósito de estimar a função sistólica e diastólica (CHETBOUL et al., 2016).

A função sistólica do VE se caracteriza por ser uma das principais avaliações utilizadas no exame ecodopplercardiográfico e, mesmo quando não é o principal foco do estudo, este índice apresenta papel importante na avaliação cardiológica, pois é um preditor no desfecho clínico nas diferentes cardiopatias (MATHIAS JUNIOR, 2018).

\section{Fração de encurtamento}

A FS foi um dos primeiros índices ecocardiográficos utilizados para avaliar a função sistólica e provavelmente ainda é a medida mais usual que pode ser obtida por diversas técnicas ecocardiográficas, contudo a janela paraesternal direita em eixo curto por modo $M$ segue sendo a de maior eleição (Figura 4) (BOON, 2011; CHETBOUL et al., 2016; SURESH, 2016).

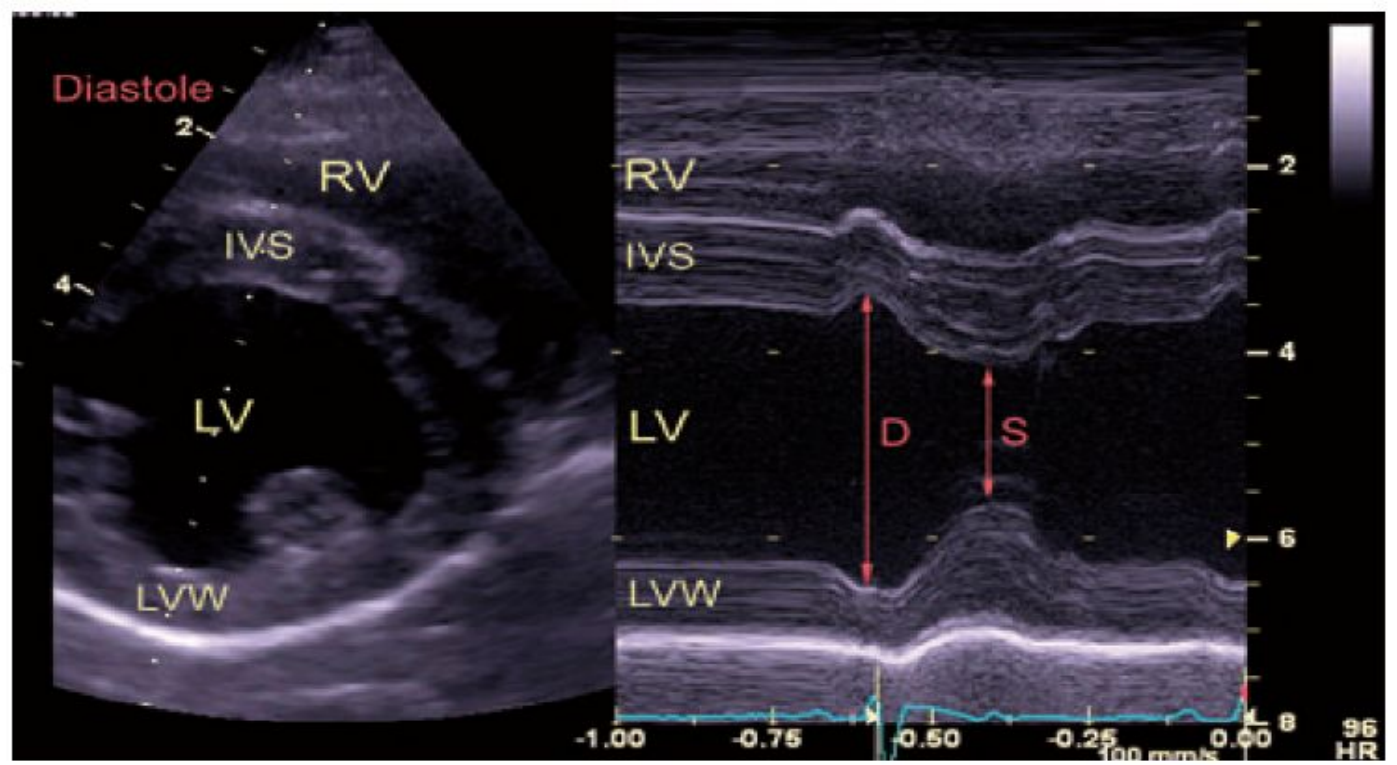

FIGURA 4 - Imagem em eixo curto do VE (esquerda) e modo M (direita) obtidos através da janela paraesternal direita. A imagem mostra as dimensões da cavidade interna do VE. Calcula-se a FS a partir dos diâmetros internos do VE nas fases de diástole (D) e sístole (S), obtendo a FS pela diferença entre os diâmetros D e $\mathrm{S}$, e após dividido por $\mathrm{D}$ e multiplicado por 100. Fonte: Bonagura e Schober (2009) 
Para obter a FS, subtrai-se o diâmetro do VE em diástole pelo diâmetro do VE em sístole e divide-se este resultado pelo diâmetro do VE em diástole, multiplicando-se o resultado por 100 para chegar a um valor percentual, conforme equação abaixo (BONAGURA; SCHOBER, 2009, BOON, 2011):

$$
F S(\%)=\frac{D V E d-D V E s}{D V E d} \times 100
$$

sendo, DVEd = diâmetro do VE em diástole; DVEs = diâmetro do VE em

sístole.

Valores de normalidade para a FS em cães variam de $34.4 \pm 6.5$, sendo que este índice em certas raças como o Doberman Pinscher poderá apresentar valores normais de FS inferiores às demais raças (CHETBOUL et al., 2016). É importante ressaltar que a FS não permite avaliar a contratilidade miocárdica diretamente, pois trata-se de um índice de função sistólica, tendo como fatores que podem afetar o resultado: a pré-carga, pós-carga e a contratilidade (BOON, 2011).

\section{Fração de ejeção}

Define-se como FE o volume ejetado pelo VE após cada ciclo cardíaco, sendo quantificado através do volume ventricular esquerdo em fim de diástole, subtraído pelo volume ventricular esquerdo em fim de sístole, dividido pelo volume ventricular esquerdo em fim de diástole, sendo expresso em percentual por meio da fórmula (BOON, 2011):

$$
F E(\%)=\frac{V V E d-V V E s}{V V E d} \times 100
$$

sendo, VVEd = volume do VE em fim de diástole; VVEs = volume do VE em fim de sístole.

Dentre os índices disponíveis para determinar a função sistólica do VE, a FE está entre os mais utilizados (BOON, 2011). Para avaliação quantitativa da FE deve-se obter valores dos volumes ventriculares em fase inicial e final do ciclo cardíaco, a partir de imagens bidimensionais ou em modo M (BONAGURA; SCHOBER, 2009). Basicamente há três métodos ecocardiográficos que podem ser utilizados para determinar o volume ventricular, que são: o método de Teicholz, também conhecido como método geométrico, e outros dois métodos planimétricos que incluem o método de Simpson e o método da área por comprimento (MATHIAS JUNIOR, 2018).

O método de Teicholz é amplamente utilizado, pois permite calcular de modo simples o volume do VE em suas diversas fases a partir do diâmetro interno da cavidade ventricular, contudo é importante reconhecer suas limitações, particularmente em cães que possuem uma geometria ventricular diferente de humanos (CHETBOUL et al., 2016). 
A equação de Teicholz conforme descrita a seguir, visa determinar o volume ventricular com o uso da fórmula do cubo utilizando uma imagem em modo $\mathrm{M}$, e pode ser aplicada quando há aumento das dimensões internas do ventrículo, porém apresenta restrições quando existem alterações na contratilidade segmentar, pois o coração perde a forma elipsoide e torna-se mais esférico (MATHIAS JUNIOR, 2018).

$$
V D f=\frac{(D d)^{3} x 7}{2,4+D d} \quad V S f=\frac{(D s)^{3} x 7}{2,4+D s}
$$

Fim de diástole $\quad$ Fim de sístole

Sendo: VDf = volume diastólico final; VSf = volume sistólico final; Dd = diâmetro do VE em fim de diástole; Ds = diâmetro do VE em fim de sístole.

Outro sistema utilizado para determinar o volume ventricular esquerdo é o método de Simpson, que apresenta maior sensibilidade que a técnica em modo $\mathrm{M}$. Este método é obtido a partir da janela paraesternal direita, eixo longitudinal quatro câmaras ou paraesternal esquerdo apical 4 câmaras ou em ambas janelas em modo modificado apenas com 2 câmaras (Figura 5) (WESS et al., 2017).

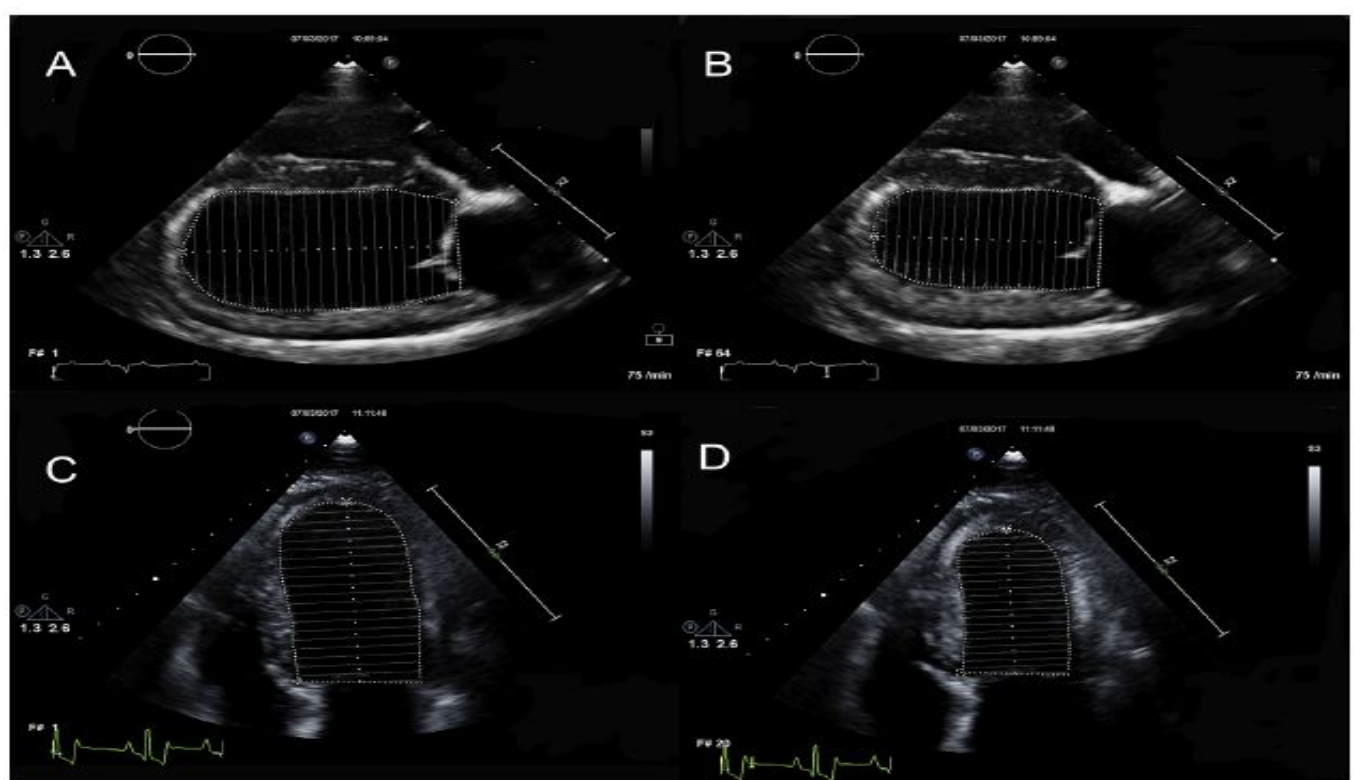

FIGURA 5 - Método de Simpson para determinar o volume ventricular esquerdo. Janela paraesternal direita, eixo longitudinal quatro câmaras em diástole $(A)$ e em sístole $(B)$. Janela paraesternal esquerda, corte apical quatro câmaras em diástole (C) e em sístole (D). O volume diastólico final (VDf) é selecionado pouco antes do inicio do complexo QRS e o volume sistólico final (VSf) selecionado no fim da onda T. Neste método o VE é fragmentado em discos e a FE é calculada para cada disco e a média total das frações isoladas de cada um determina a FE global. Fonte: Wess et al. (2017) 
Por este método, a área interna do VE é calculada a partir do contorno realizado nas bordas endocárdicas, dividindo esta área em discos, sendo que o volume total do VE é calculado a partir da soma dos discos ou volumes, assim o valor da FE é realizado para cada segmento isoladamente e a média total das frações isoladas de cada um representa a FE global do VE (CHETBOUL et al., 2016; MATHIAS JUNIOR, 2018).

Também é possível determinar o volume ventricular baseado na áreacomprimento desta cavidade, sendo obtidas imagens a partir da janela paraesternal esquerda apical 4 câmaras, em seguida traçando uma área a partir dos bordos endocárdicos nas fases sistólica e diastólica, e após, determina-se o diâmetro interno do ventrículo esquerdo entre a base e o ápice (Figura 6) (BONAGURA; SCHOBER, 2009; BOON, 2011; CHETBOUL et al., 2016).

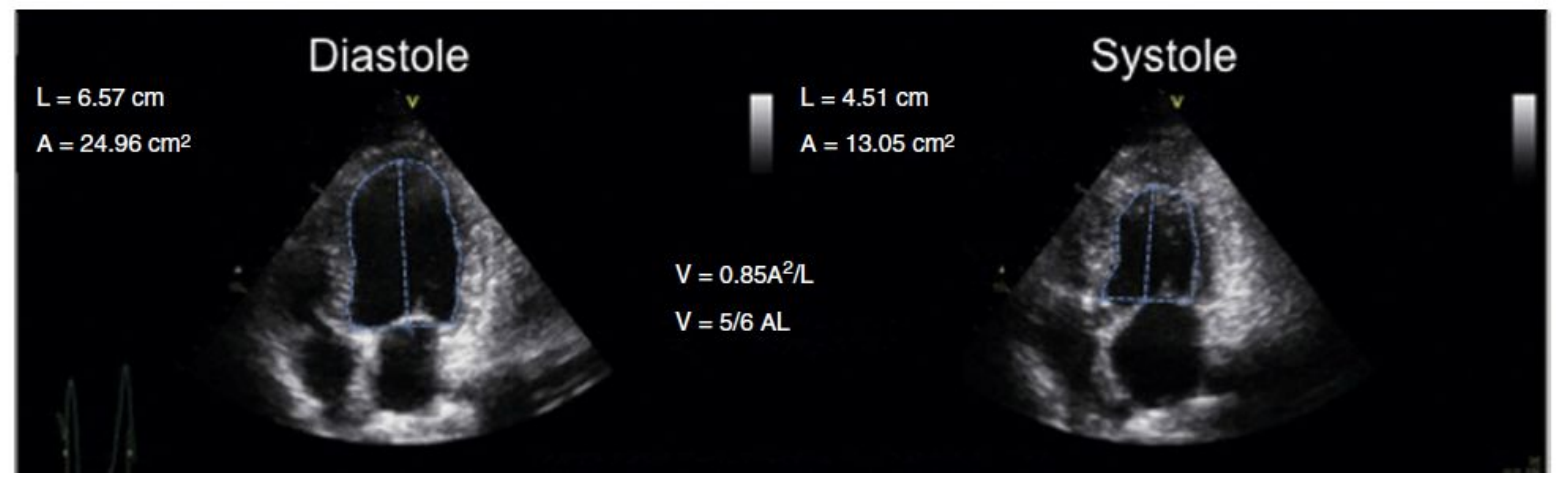

FIGURA 6 - Medidas de volumes diastólicos e sistólicos por método de áreacomprimento. O volume do VE em diástole é obtido pelo produto da área interna do VE ao quadrado divido pelo comprimento do ápice cardíaco ao nível da valva mitral, sendo realizado o mesmo procedimento na fase sistólica.

Fonte: Chetboul et al. (2016)

Várias são as limitações e dificuldades em obter critérios de função sistólica, tendo como principal dificuldade o manejo técnico, como obter uma boa janela ecocardiográfica com perfeita visualização dos bordos endocárdicos ventriculares e entender os fatores que influenciam os resultados da função sistólica (CHETBOUL et al., 2016). Os valores de normalidade para a FE podem variar de acordo com a técnica utilizada para obter este índice conforme a tabela 1 (CHETBOUL et al., 2016).

TABELA 1 - Valores de normalidade para a FE para cães, calculados por diferentes métodos.

\begin{tabular}{|c|c|c|c|}
\hline Método & Simpson & $\begin{array}{c}\text { Área } x \\
\text { comprimento }\end{array}$ & Teicholz \\
\hline FE (\%) & $66.5 \pm 6.4$ & $65.5 \pm 6.7$ & $70.9 \pm 6.0$ \\
\hline
\end{tabular}

FONTE: Adaptado de Chetboul et al. (2016) 
Dentre as técnicas apresentadas para determinar o volume ventricular, está recomendada pela Sociedade Americana de Ecocardiografia a do método de Simpson (BOON, 2011).

\section{Movimento do Anel Mitral}

Vários outros índices são propostos para avaliar a função sistólica antes que as fibras radiais sejam afetadas, dentre estes o movimento do anel mitral (MAM) obtido pelo modo M ou doppler tecidual (Figura 7) (CHETBOUL et al., 2016; SPALLA et al., 2018).

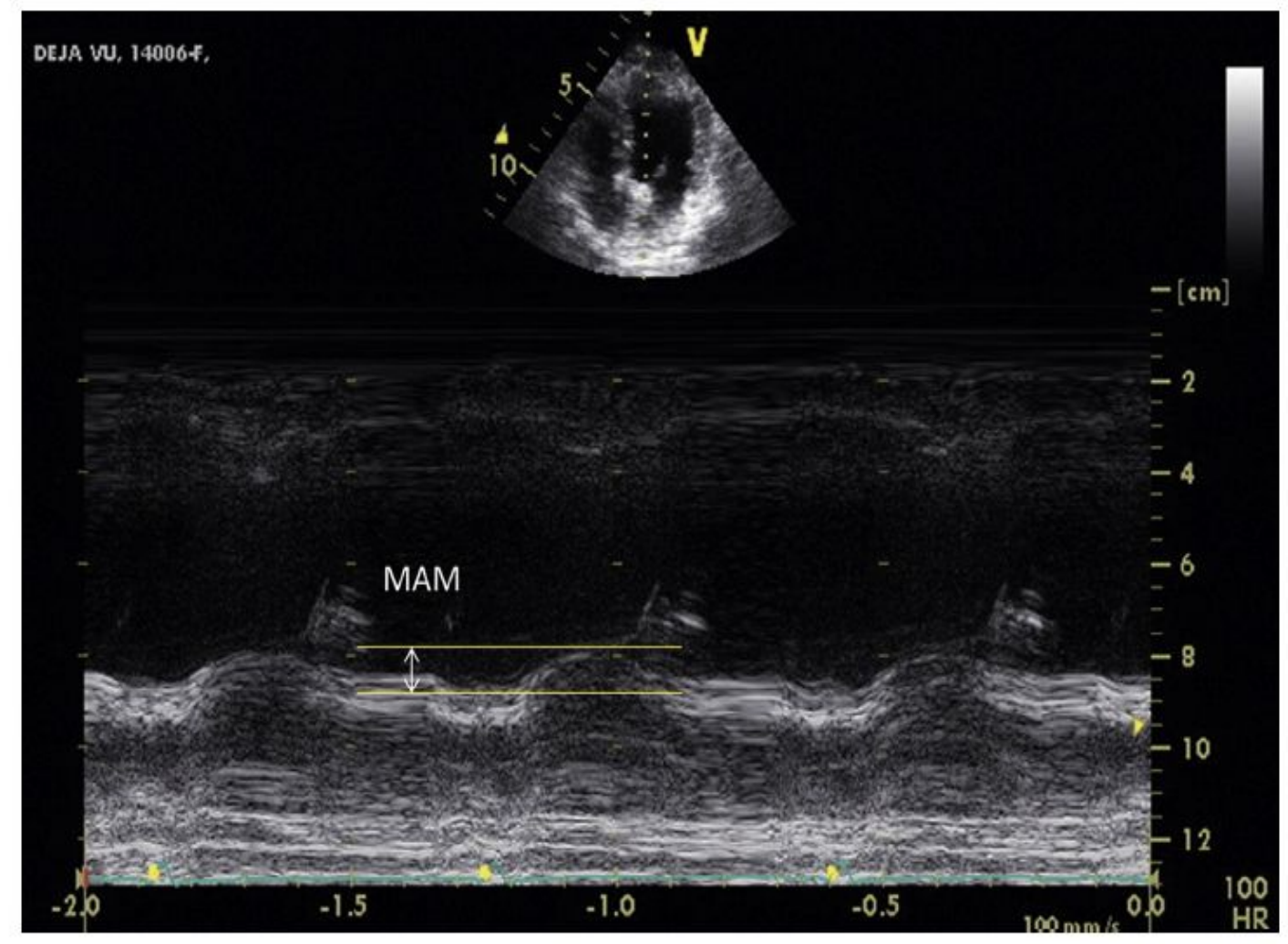

FIGURA 7 - Imagem ecocardiográfica que ilustra como obter a medida do movimento do anel mitral (MAM) a partir da janela paraesternal esquerda apical quatro câmaras a partir da linha em modo M. Esta medida é obtida a partir do deslocamento máximo do anel mitral em direção ao ápice cardíaco (retas).

Fonte: Chetboul et al. (2016)

As fibras miocárdicas longitudinais são descritas como as primeiras estruturas a serem prejudicadas quando se inicia o processo de disfunção ventricular sistólica, assim a avaliação ecocardiográfica longitudinal pode auxiliar no diagnóstico da disfunção ventricular em tempo mais precoce (WOLF et al., 2018).

O MAM correlaciona diretamente com a FE, e pode ser obtido através da janela paraesternal esquerda apical com o uso do modo $\mathrm{M}$ com o cursor situado sobre o anel mitral septal. Ressalta-se que o movimento gerado pelo anel mitral 
tende a diminuir com a idade, contudo apenas nos pacientes com FE normais, enquanto que a idade não influi no resultado do MAM em pacientes com FE reduzidas (BOON, 2011).

Em cães normais o MAM pode variar de 0,46 a $1,74 \mathrm{~cm}$, sendo que este índice apresenta forte relação com o peso corpóreo do animal. Animais com peso inferior a $15 \mathrm{~kg}$ tem um intervalo de confiança de 95\% para MAM com valores de 0,65 a $0,75 \mathrm{~cm}$. Já para cães com peso corpóreo variando entre $15 \mathrm{~kg}$ a $40 \mathrm{~kg} 0$ mesmo intervalo de confiança de $95 \%$ são para valores de 1,03 a $1,13 \mathrm{~cm}$. Por fim, cães acima de $40 \mathrm{~kg}$ apresentam valores de normalidade entre 1,21 a 1,81 cm (BOON, 2011).

O MAM pode ser indexado através da superfície corpórea a partir do encurtamento das fibras radiais e longitudinais do VE, expresso pela seguinte fórmula (BOON, 2011; CHETBOUL et al., 2016):

$$
\operatorname{MAM}(\%)=\frac{M A M}{(V E d-V E s)+M A M} \times 100
$$

Sendo: VEd = diâmetro do ventrículo esquerdo em diástole; VEs = diâmetro do ventrículo esquerdo em sístole.

Os valores de normalidade para o MAM em cães estão descritos conforme a tabela 2:

TABELA 2 - Valores de normalidade para o MAM em cães.

\begin{tabular}{|l|c|c|c|}
\hline & $<15 \mathrm{~kg}$ & $\geq 15 \mathrm{~kg} \mathrm{e} \leq 40 \mathrm{~kg}$ & $>40 \mathrm{~kg}$ \\
\hline MAM $(\mathrm{cm})$ & $0.70(0.65-0.75)$ & $1.08(1.03-1.13)$ & $1.51(1.21-1.81)$ \\
\hline MAM \% & $40(38-42)$ & $42(40-44)$ & $44(39-49)$ \\
\hline
\end{tabular}

FONTE: Adaptado de Chetboul et al. (2016)

\section{Índice de Performance Miocárdica}

A maior parte dos pacientes cardiopatas em fase de IC, costumam desenvolver simultaneamente disfunção ventricular sistólica e diastólica, o que impacta diretamente no diagnóstico, prognóstico e tratamento (ALVAREZ et al., 2010).

O índice de performance miocárdica (IPM) ou comumente conhecido como índice de Tei é uma medida de função miocárdica global que inclui simultaneamente avaliação da função sistólica e diastólica, sendo que em humanos este índice tem sido utilizado para fornecer informações prognósticas em pacientes com estenose valvar aórtica, cardiomiopatia dilatada e regurgitação mitral (BOON, 2011).

Em cães o IPM tem demonstrado sua importância diagnóstica em animais com cardiomiopatia dilatada em fase subclínica (ALVAREZ et al., 2010; BOON, 2011; CHETBOUL et al., 2016). Este índice é obtido com o uso do doppler a partir do fluxo transvalvar mitral e fluxo aórtico a partir da janela paraesternal esquerda apical quatro e cinco câmaras, que consiste na soma do tempo de contração 
isovolumétrica (TCI) com o tempo de relaxamento isovolumétrico (TRIV), divididos pelo tempo de ejeção ventricular (TE) (Figura 8) (ALVAREZ et al., 2010).
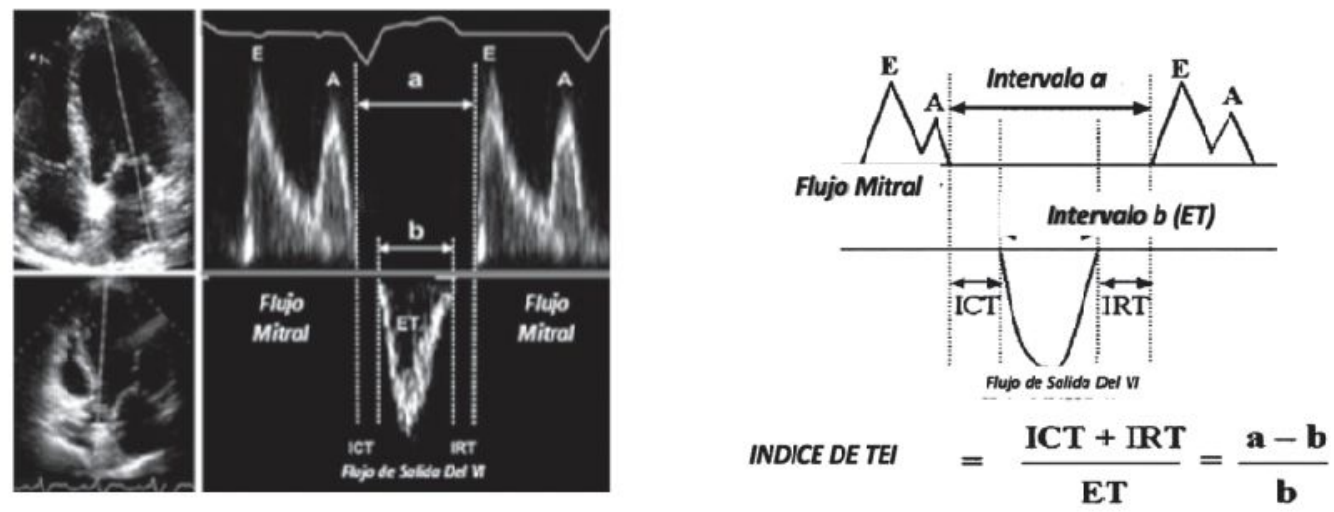

FIGURA 8 - Registro ecocardiográfico de TEI. A imagem a esquerda obtida pela janela paraesternal esquerda apical quatro câmaras, mostra o fluxo transmitral. O fluxo aórtico obtém do trato de saída do ventrículo esquerdo. À direita o esquema gráfico do índice de TEl, e demonstração das seguintes medidas para realizar o cálculo. ICT ou TCl: tempo de contração isovolumêtrica; IRT ou TRIV: tempo de relaxamento isovolumêtrico; ET: tempo de ejeção ventricular. $O$ índice de TEI é calculado pela soma do TCI com o TRIV e após dividido pelo ET.

Fonte: Adaptado de Álvarez et al. (2010)

Em um estudo realizado com cães da raça Newfoundland, foram estudados animais, com e sem cardiomiopatia dilatada (CMD), e avaliou-se o uso do IPM nestes pacientes, demonstrando ser um índice que não sofre influência de alguns fatores como idade, sexo, área de superfície corpórea e frequência cardíaca, assim, o IPM confirmou ser um índice ecocardiográfico diretamente relacionado a disfunção sistólica (LEE et al., 2002). O valor de normalidade para o índice de TEI em cães saudáveis é de $0.33 \pm 0.10$ (ALVAREZ et al., 2010).

\section{$\mathrm{dP} / \mathrm{dT}$}

A mudança no gradiente de pressão sistólica entre ventrículo esquerdo e átrio esquerdo com o decorrer do tempo é determinada considerando o intervalo necessário para a velocidade do jato regurgitante aumentar a sua velocidade de 1 para $3 \mathrm{~m} / \mathrm{s}$ ou de 4 para $36 \mathrm{mmHg}$ calculado pela equação modificada de Bernoulli (MATHIAS JUNIOR, 2018).

A dP/dT é um cálculo não invasivo de pressão e tempo que representa uma medida indireta da contratilidade miocárdica, utilizado para avaliar a função ventricular esquerda através da regurgitação mitral, que por derivação a mudança de pressão do ventrículo esquerdo, permite estimar a função global do ventrículo esquerdo, pois este índice tende a diminuir em pacientes com insuficiência valvar mitral avançada como consequência da disfunção ventricular (BONAGURA; SCHOBER, 2009). 
Estudo por telemetria em animais provou que a dP/dT é um índice com alta sensibilidade na avaliação da contratilidade miocárdica, sendo que cães normais tendem a apresentar valores de $\mathrm{dP} / \mathrm{dT}$ superiores a $1800 \mathrm{mmHg} / \mathrm{s}$ (Figura 9) (BUCHANAN et al., 2016).

(a)

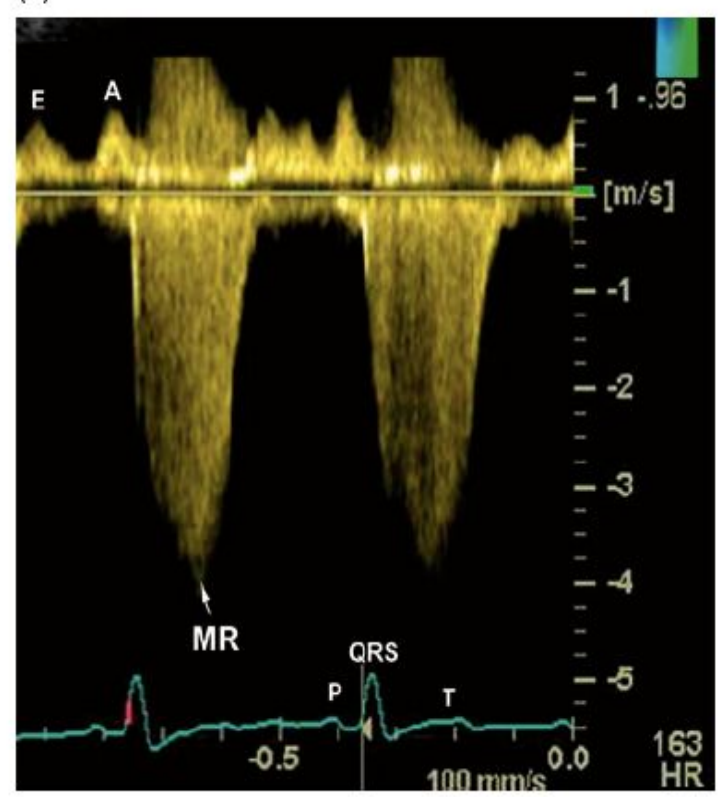

(b)

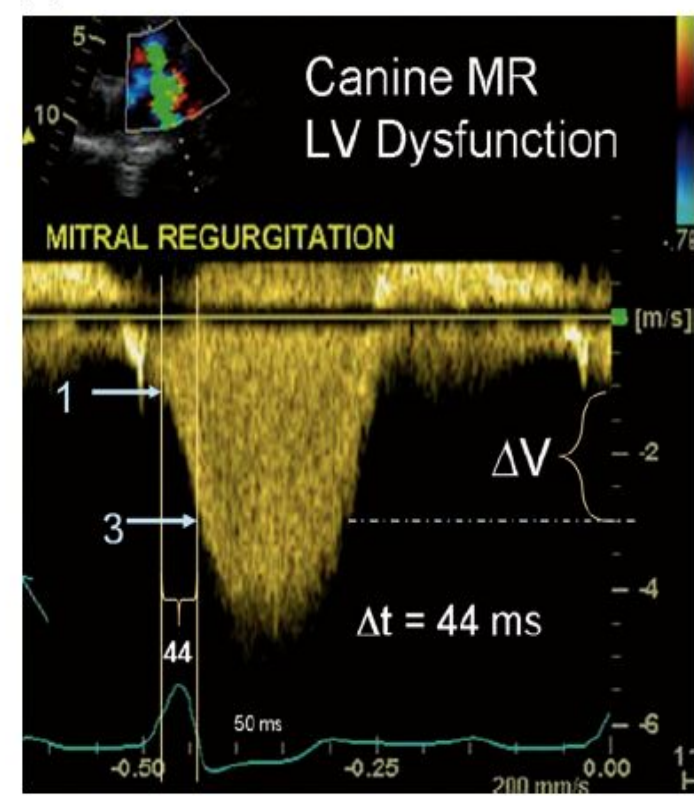

FIGURA 9 - a) Registro espectral da insuficiência valvar mitral através do doppler contínuo (CW). b) Método para cálculo da dP/dT através da regurgitação mitral. Observar que foi calculado o tempo entre os pontos 1 e $3 \mathrm{~m} / \mathrm{s}$ com resultado de $44 \mathrm{~ms}$. Após obter a distancia entre os pontos 1 e 3 calcula-se a $\mathrm{dP} / \mathrm{dT}$ divindo $32 \mathrm{mmHg}$ pelo intervalo obtido.

Fonte: Bonagura e Schober (2009)

Uma vez obtido o valor do intervalo Delta T, procede com o cálculo da $\mathrm{dP} / \mathrm{dT}$ que deve ser realizado da seguinte forma:

$$
d P / d T=\frac{32 m m H g}{\text { intervalo }(\text { seg })}
$$

\section{Separação septal do ponto E}

A separação entre o ponto $E$ e o septo interventricular (EPSS) é uma medida realizada através do modo $\mathrm{M}$ que correlaciona diretamente com o diâmetro do VE. Sabendo que a disfunção sistólica tende a progredir com a dilatação do VE e com a diminuição do fluxo transmitral, ocorrerá um aumento na distância entre o ponto $\mathrm{E}$ ou ponto de maior abertura da válvula mitral septal com o septo interventricular (SIV) (Figura 10) (MCKAIGNEY et al., 2014; HOLLER; WESS, 2014). 


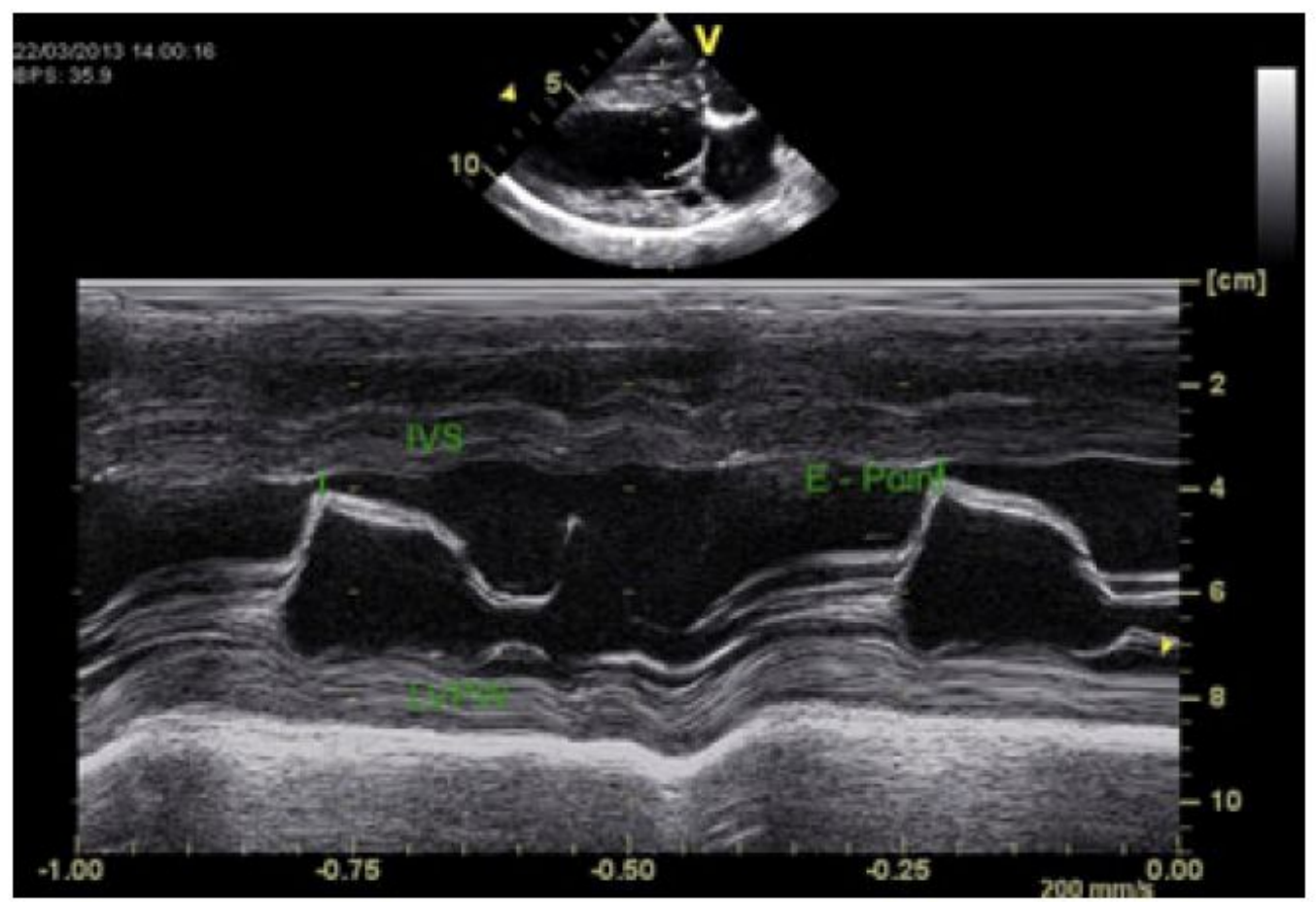

FIGURA 10 - Modo-M realizado a partir da figura 8. A linha na vertical mostra a distância entre o movimento de máxima abertura da da válvula mitral septal ao SIV durante a fase de enchimento ventricular rápido, distância esta que representa o EPSS.

FONTE: Holler e Wess (2014)

O EPSS é um parâmetro utilizado para avaliação do VE durante as fases de enchimento e ejeção e com forte correlação negativa com a FE em pacientes que não apresentam insuficiência das valvas aórticas e mitral, sendo que na presença destas o EPSS não deve ser utilizado como índice de função sistólica devido as alterações hemodinâmicas e na contratilidade cardíaca (HOLLER; WESS, 2014).

\section{Strain e Strain Rate}

ST corresponde à deformação miocárdica, pois permite calcular o percentual de mudança no comprimento de um determinado segmento do miocárdio. Já o STR refere-se a taxa de mudança do ST, ou seja, a velocidade com que ocorre a deformação (DEL CASTILLO, 2013).

O exame ecodopplercardiográfico com o uso da tecnologia doppler em modo bidimensional Speckle Tracking (2D-STE), é considerado como uma das mais novas ferramentas disponíveis na rotina clínica e no campo experimental para quantificar a função miocárdica segmentar, que tem demonstrado oferecer mais vantagens quando comparado à ecocardiografia convencional (TOALDO et al., 2017). Esta técnica é baseada na análise do deslocamento espacial dos cardiomiócitos (tracking) das manchas digitais (speckle) geradas através da interação entre o feixe de ultrassom e as fibras miocárdicas durante o exame bidimensional (MONDILLO et al., 2011).

$\mathrm{Na}$ imagem ecocardiográfica estes speckles aparecem como pequenos elementos brilhantes no miocárdio e atuam como marcadores acústicos teciduais 
naturais que podem ser monitorados em modo contínuo durante todas as fases do ciclo cardíaco (CHETBOUL et al., 2016).

As variáveis ST e STR obtidos por meio do 2D-STE é um método já estabelecido em humanos, e os resultados apresentam valores semelhantes quando comparados a ressonância magnética (Figura 11) (BONAGURA; SCHOBER, 2009).

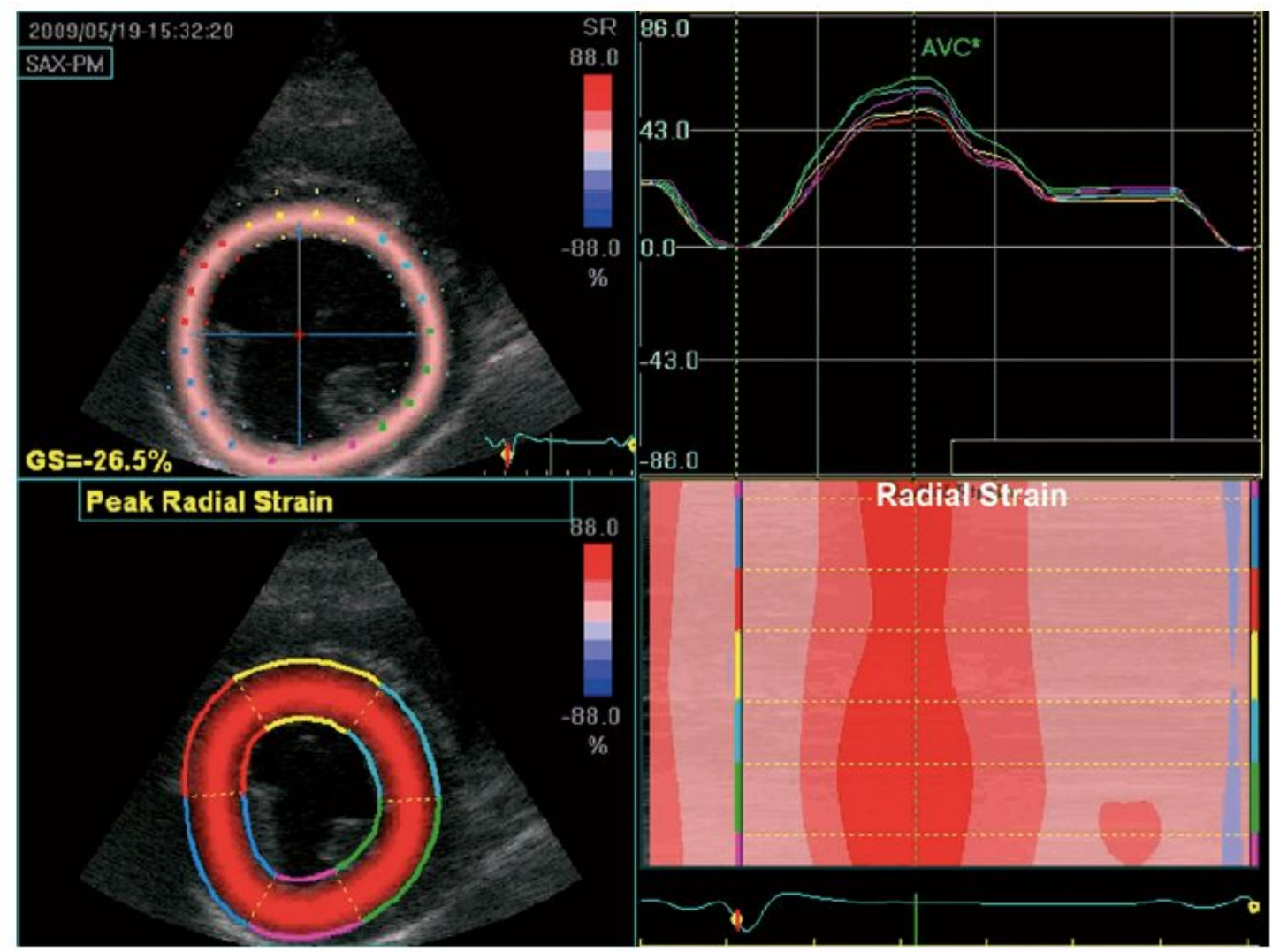

FIGURA 11 - Tensão radial segmentar realizada em um cão com insuficiência mitral com algoritmos 2D-STE. O painel superior esquerdo mostra seis setores arbitrários no início da sístole antes da ejeção. A parte inferior esquerda mostra o pico radial codificado por cores tensão (deformação) atingida no final da sístole. As alterações codificadas por cores mostram que a deformação ocorreu de forma sincrônica, atingindo o pico próximo da sístole. Os valores reais são mostrados no gráfico superior direito.

Fonte: Bonagura e Schober (2009)

Esta técnica tem demonstrado ser de grande valia para o estudo da função ventricular, permitindo diagnóstico de maneira precoce de algumas miocardiopatias, tanto em humanos quanto em pequenos animais (WESS et al., 2010).

Apesar dos recursos de ST e STR atuarem como índices que permitem o diagnóstico precoce da disfunção ventricular, a aplicação clínica para pequenos animais requer mais pesquisas antes que sejam utilizados na decisão clínica em medicina veterinária (BONAGURA; SCHOBER, 2009). 


\section{CONSIDERAÇÕES FINAIS}

A ecocardiografia com a modalidade doppler é uma das principais ferramentas diagnóstica para avaliação cardíaca pois permite, por técnica não invasiva, adquirir imagens das estruturas cardíacas e avaliar a função miocárdica. $\mathrm{O}$ uso correto deste exame complementar proporciona ao médico veterinário ecocardiografista caracterizar a função ventricular sistólica e diastólica, regional e global, o que aumenta a possibilidade de diagnóstico das cardiopatias e o acompanhamento da evolução no tratamento do paciente cardiopata. Com o avanço tecnológico em equipamentos de alta qualidade e na grande variedade técnicas ecodopplercardiográficas disponíveis, permitiram melhor acurácia na avaliação da função ventricular esquerda minimizando erros na avaliação quantitativa, contudo ainda faltam estudos que permitem um diagnóstico precoce da disfunção ventricular em medicina veterinária quando aplicado a técnicas não convencionais.

\section{REFERÊNCIAS}

ALVAREZ, I.; ORTIZ, C.; CRUZ, L. E. Determinación de los valores del índice de TEI (índice de performance miocárdico) en caninos con insuficiencia cardiaca, evaluados mediante ecocardiografia doppler. Revista de Medicina Veterinaria. n. 20, p. 81-94, 2010. Disponível em: <http://www.scielo.org.co/pdf/rmv/n20/n20a08.pdf>

BONAGURA, J. D.; SCHOBER, K. E. Can ventricular function be assessed by echocardiography in chronic canine mitral valve disease?. Journal of Small Animal Practice. 2009. Disponível em: <https://doi.org/10.1111/j.1748-5827.2009.00803.x>. doi: 10.1111/j.1748-5827.2009.00803.x

BOON, J. A. Evaluation of size, function, and hemodynamics. In: Veterinary echocardiography. 2 ed. New Jersey: John Willey; p. 151-260, 2011.

BUCHANAN, L. V.; WARNER, W. A.; ARTHUR, S. R.; GLEASON, C. R.; LEWEN, G. et al,. Evaluation of cardiac function in unrestrained dogs and monkeys using left ventricular dP/dt. Journal of Pharmacological and Toxicological Methods. n. 80, p. 51-58, 2016. Disponível em: < https://doi.org/10.1016/j.vascn.2016.03.006>. doi: 10.1016/j.vascn.2016.03.006

CHETBOUL, V.; BUSSADORI, C.; MADRON, E. Clinical echocardiography of the dog and cat. 1 ed. St. Louis: Elsevier; 2016.

DEL CASTILLO, J. M. Strain Cardíaco. 1 ed. Rio de Janeiro: Revinter; 2013.

HOLLER, P. J.; WESS, G. Sphericity índex E-point-to-septal-separation (EPSS) to diagnose dilated cardiomyopathy in Doberman Pinschers. Journal of Veterinary Internal Medicine. n. 28, p. 123-129, 2014. Disponível em: <https://doi.org/10.1111/jvim.12242>. doi: 10.1111/jvim.12242

KLABUNDE, R. E. Cardiovascular physiology concepts. 2 ed. Baltimore: Lippincott Williams \& Wilkins; 2012. 
LAVALLE, R.; RENDON, L. Estructuración y función del miocárdio ventricular canino (Canis lupus familiaris): banda miocárdica ventricular. Spei Domus. v. 22, p. 25-35, 2015. Disponível em: <https://doi.org/10.16925/sp.v11i22.1156>. doi: 10.16925/sp.v11i22.1156

LEE, B. H.; DUKES MC-EWAN, J.; FRENCH, A. T.; CORCORAN, B. M. Evaluation of a novel doppler índex of combined systolic and diastolic myocardial performance in Newfoundland dogs with familial prevalence of dilated cardiomyopathy. Veterinary Radiology and Ultrasound. n. 43, p. 154-165, 2002. Disponível em: < https://doi.org/10.1111/j.1740-8261.2002.tb01663.x>. doi: 10.1111/j.17408261.2002.tb01663.x

MATHIAS JUNIOR, W. Ecocardiografia para o dia a dia. 1 ed. Barureri: Manole; 2018.

MCKAIGNEY, C. J.; KRANTZ, M. J.; LA ROCQUE, C. L.; HURST, N. D.; BUCHANAN, M. S. et al,. E-point septal separation: a bedside tool for emergency physician assessment of left ventricular ejection fraction. The American Journal of Emergency Medicine. n. 32, p. 493-497, 2014. Disponível em: < https://doi.org/10.1016/j.ajem.2014.01.045>. doi: 10.1016/j.ajem.2014.01.045

MONDILLO, S.; GALDERISI, M.; MELE, D.; CAMELI, M.; LOMORIELLO, V. S. et al,. Speckle-tracking echocardiography: a new technique for assessing myocardial function. Journal of Ultrassound in Medicine. v. 30, p. 71-83, 2011. Disponível em: < https://doi.org/10.7863/jum.2011.30.1.71>. doi: 10.7863/jum.2011.30.1.71

POLLOCK, J. D.; MAKARYUS, A. N. Physiology, Cardiac Cycle. In: StatPearls. $\begin{array}{llll}\text { Treasure Island 2018. Disponível } & \text { (FL). }\end{array}$ https://www.ncbi.nlm.nih.gov/books/NBK459327/

SALAMANCA, D. R.; BERNAL-GARCÍA, M.; CASTRO, I. D. Disección experimental de la banda miocárdica ventricular. Revista de Investigación en Salud. Universidad de Boyacá. v. 2, n. 2, p. 148-161, 2015. Disponível em: $<$ https://www.researchgate.net/publication/317424221_Diseccion_experimental_de_I a_banda_miocardica_ventricular>. doi: 10.24267/23897325.134

SARGENT, J.; MUZZI, R.; MUKHERJEE, R.; SOMARATHNE, S.; SCHRANZ, K. et al. . Echocardiography predictors of survival in dogs with myxomatous mitral valve disease. Journal of Veterinary Cardiology. v. 1, p. 1-12, 2015. Disponível em: $<$ https://doi.org/10.1016/j.jvc.2014.11.001>. doi: 10.1016/j.jvc.2014.11.001

SPALLA, L.; PAYNE, J. R.; BORGEAT, K.; FUENTES, L. V.; CONNOLLY, D. J. Prognostic value of mitral annular systolic plane excursion and tricúspide annular plane systolic excursion in cats with hypertrophic cardiomyopathy. Journal of Veterinary Cardiology. n. 20, p. 154-164, 2018. Disponível em: < https://doi.org/10.1016/j.jvc.2018.04.005>. doi: 10.1016/j.jvc.2018.04.005 
SURESH, C. Left ventricular global systolic function assessment by echocardiography. Annals of Cardiac Anaesthesia. n. 19, p. 26-34, 2016. Disponível em: <https://dx.doi.org/10.4103\%2F0971-9784.192617> doi: 10.4103\%2F0971-9784.192617

TOALDO, M. B.; ROMITO, G., GUGLIELMINI, C.; DIANA, A.; PELLE, N. G. et al,. Assessment of Left Atrial Deformation and Function by 2-Dimensional Speckle Tracking Echocardiography in Healthy Dogs and Dogs with Myxomatous Mitral Valve Disease. Journal of Veterinary Internal Medicine. n.31, p. 641-649, 2017. Disponível em: https://dx.doi.org/10.1111\%2Fjvim.14722

WESS, G.; HABIL, D. O.; DUKES-MCEWAN, J.; HAGGSTROM, J.; GORDON, S. European society of veterinary cardiology screening guidelines for dilated cardiomiopathy in doberman pinschers. Journal of Veterinary Cardiology. n. 19, p. 405-415, 2017. Disponível em: <https://doi.org/10.1016/j.jvc.2017.08.006>. doi: 10.1016/j.jvc.2017.08.006

WESS, G.; SARKAR, R.; HARTMANN, K. Assessment of left ventricular systolic function by strain imaging echocardiography in various stages of feline hypertrophic cardiomyopathy. Journal of Veterinary Internal Medicine. v. 24, p. 1375-1382, 2010. Disponível em: < https://doi.org/10.1111/j.1939-1676.2010.0586.x>. doi: 10.1111/j.1939-1676.2010.0586.x

WOLF, M.; LUCINA, S. B.; BRULER, B. C.; TULESKI, G. L. R.; SILVA, V. B. C., et al. Assessment of longitudinal systolic function using tissue motion annular displacement in healthy dogs. Journal of Veterinary Cardiology. n. 20 p. 175-185, 2018. Disponível em: < https://doi.org/10.1016/j.jvc.2018.04.004>. doi: 10.1016/j.jvc.2018.04.004 